\title{
Continuation Semantics for PROLOG with Cut
}

\author{
A. de Bruin \\ Faculty of Economics, Erasmus University \\ P.O.Box 1738, 3000 DR Rotterdam, the Netherlands \\ E.P. de Vink \\ Department of Mathematics and Computer Science, Free University \\ De Boelelaan 1081, $1081 \mathrm{HV}$ Amsterdam, the Netherlands
}

\begin{abstract}
We present a denotational continuation semantics for PROLOG with cut. First a uniform language $\mathscr{B}$ is studied, which captures the control flow aspects of PROLOG. The denotational semantics for $\mathscr{B}$ is proven equivalent to a transition system based operational semantics. The congruence proof relies on the representation of the operational semantics as a chain of approximations and on a convenient induction principle. Finally, we interpret the abstract language $\mathscr{B}$ such that we obtain equivalent denotational and operational models for PROLOG itself.
\end{abstract}

\section{Section 1 Introduction}

In the nice textbook of Lloyd on the foundations of logic programming [Ll] the cut, available in all PROLOG systems, is described as a controversial control facility. The cut, added to the Horn clause logic for efficiency reasons, affects the completeness of the refutation procedure. Therefore the standard declarative semantics using Herbrand models does not adequately capture the computational aspects of the PROLOG language. In the present paper we study the PROLOG cut operator in a sequential environment augmented with backtracking. Our aim is to provide a denotational semantics for PROLOG with cut and to prove this semantics equivalent to an operational one.

First of all we separate the "logic programming" details (such as most general unifiers and renaming indices) in PROLOG from the specification of the flow of control, (e.g. backtracking, the cut operator). This is achieved by extracting the uniform language $\mathscr{B}$ from PROLOG - uniform in the sense of [BKMOZ] - which contains only the latter issues. Fitting within the "Logic Programming without Logic" approach, ([Ba2]), our denotational model developed for the abstract backtracking language has enough flexibility for further elaboration to a non-uniform denotational model of PROLOG itself. Moreover, the equivalence of this denotational semantics and an operational semantics for PROLOG is a straightforward generalization for the congruence proof of $\mathscr{B}$.

Secondly, our denotational semantics uses continuations. This has several advantages over earlier semantics which (essentially) are based on a direct approach. (See [Br] for a discussion on the relative merits of continuations vs. direct semantics.) We arrive at a concise set of semantical 
equations in which there is no need for coding up the states using cut flags or special tokens (as in [JM], [DM], [Vi], [Bd]). Moreover, since operational semantics - being a goal stacking model must contain (syntactical) continuations, congruence of the two semantics can be established much more elegantly.

Our final contribution can be found in the equivalence proof itself. The equivalence proof does not split - as usual - into $\mathscr{O} \subseteq \mathscr{D}$ and $\mathscr{D} \subseteq \mathscr{O}$. Rather, both the operational and denotational semantics are represented as least upperbounds of chains and we prove equality of the approximating elements. (See also [KR], [BM] where - although not made explicit - in the setting of complete metric spaces operational and denotational semantics can be represented as limits of Cauchy sequences.)

Related work on the comparative semantics of PROLOG with cut includes [JM], [DM], [Vi]. Jones \& Mycroft present a direct Scott-Strachey style denotational semantics. They do not compare this semantics with an operational one. Instead, correctness of their semantics comes from its systematic construction. In [Vi] also a direct denotational model is developed and additionally proven correct w.r.t. a transition based operational meaning. The proof is rather involved, since the cut is modeled by a special token (as in [JM]). The semantics of Debray \& Mishra is a mixture of a direct and continuation semantics. They (need to) have sequences of answers substitutions together with cut flags in their semantics. The denotational semantics is related to an operational one. However, it is not clear to us what makes their equivalence proof work. (In particular we do not understand the proof of theorem 4.1, case 5 in the appendix of [DM].) The semantics mentioned above all denote a program by a sequence of substitutions. In the present paper we only deliver the first one. This does not give rise to loss of generality, since our semantics allows extension to streams of substitutions, (as in [Vi], [Bd]). We have chosen not to do so for reasons of space and clarity of the presentation.

Acknowledgments. Our appreciation is due to Jaco de Bakker, Frank de Boer, Joost Kok, John-Jules Meyer and Jan Rutten, members of the Amsterdam Concurrency Group, who offered us a stimulating forum. We thank Aart Middeldorp and the referees for reading the manuscript. We are indebted to $\mathrm{M} 279$ for the hospitality the authors received during the preparation of this paper.

\section{Section 2 Deterministic Transition Systems}

In this section we introduce the notion of a transition system, ([P $],[\mathrm{BMOZ}]$ ). For reasons of space we restrict ourselves to deterministic transition systems, which already suit our purposes. Collections of transition systems are turned into a cpo s.t. associating a valuation to a transition system becomes a continuous operation.

(2.1) Definition A deterministic transition system $T$ is a seven tuple $\langle C, I, F, \Omega, D, \alpha, S\rangle$ where the set configurations $C$ is the disjoint union of $I, F$ and $\{\Omega\}, I$ is a set of internal configurations, $F$ is a set of final configurations, $\Omega$ is the undefined configuration, $D$ is a domain of values, $\alpha: F \rightarrow D$ is a valuation assigning a value to each final configuration and $S$ is a deterministic step or transition relation, i.e. a partial function $S: C \rightarrow_{\text {part }} C$ with $\operatorname{dom}(S) \subseteq I$. 
Next we show how to extend the valuation $\alpha$ on final configurations to a valuation $\alpha_{T}$ on arbitrary configurations of a transition system $T$.

(2.2) DefinItION Let $T=\langle C, I, F, \Omega, D, \alpha, S\rangle$ be a deterministic transition system. Denote by $D_{\perp}$ the flat cpo generated by $D$ with least element $\perp$. We associate with $T$ a mapping $\alpha_{T}$ : $C \rightarrow D_{\perp}$ defined as the least function in $C \rightarrow D_{\perp}$ s.t. $\alpha_{T}(\Omega)=\perp, \alpha_{T}(c)=\alpha(c)$ if $c \in F$, $\alpha_{T}(c)=\alpha_{T}\left(c^{\prime}\right)$ if $\left(c, c^{\prime}\right) \in S$ and $\alpha_{T}(c)=\perp$ otherwise.

Fix sets $I$ and $F$ of internal and final configurations, respectively. Fix an undefined configuration $\Omega$, a domain of values $D$, a valuation function $\alpha: F \rightarrow D$ and put $C=I \cup F \cup\{\Omega\}$. Let $T S=\left\{\langle C, I, F, \Omega, D, \alpha, S\rangle \mid S: C \rightarrow_{\text {part }} C\right.$ with $\left.\operatorname{dom}(S) \subseteq I\right\}$ denote the collection of all deterministic transition systems with configurations in $C$, internal configurations in $I$, final configurations in $F$, undefined configuration $\Omega$, domain of values $D$ and valuation function $\alpha$. In $T S$ we identify a transition system with its transition relation. (In particular we may write $T(c)$ and $c \rightarrow T c^{\prime}$ rather than $S(c)$ or $\left(c, c^{\prime}\right) \in S$ for a transition system $T$ with step relation $S$.)

We consider the set of configurations as a flat cpo with ordering $\leqq c$ and least element $\Omega$. This induces an ordering $\leqq T S$ on $T S$ as follows: $T_{1} \leqq T S T_{2} \Leftrightarrow \operatorname{dom}\left(T_{1}\right) \leqq \operatorname{dom}\left(T_{2}\right)$ \& $\forall c \in \operatorname{dom}\left(T_{1}\right): T_{1}(c) \leqq c T_{2}(c)$. We have that $T S$ is a cpo when ordered by $\leqq r S$. (The nowhere defined transition system $\varnothing$ is the least element of $T S$; for a chain $\left\langle T_{k}\right\rangle_{k}$ in $T S$ the transition system $T$ with $\operatorname{dom}(T)=U_{k} \operatorname{dom}\left(T_{k}\right)$ and $T(c)=l u b_{k} T_{k}(c)$ acts as least upperbound.) Moreover, the operation $\lambda T . \alpha_{T}: T S \rightarrow C \rightarrow D_{\perp}$ that assigns to a transition system the valuation it induces, is continuous with respect to $\leqq T S$. (See [Vi].)

REMARK Let $I_{0} \leqq I_{1} \leqq \cdots$ be an infinite sequence of subsets of internal configurations s.t. $I=\cup_{k} I_{k}$. Put $C_{k}=I_{k} \cup F \cup\{\Omega\}$. Then we can construct for each $T \in T S$ a chain of approximations $\left\langle T_{k}\right\rangle_{k}$ of $T$ in $T S$, where $T_{k}$ is defined as the smallest deterministic transition system s.t. $T_{k}(c)=T(c)$ if $c \in I_{k}, T(c) \in C_{k}$, and $T_{k}(c)=\Omega$ if $c \in I_{k} \& T(c)$ is defined but $T(c) \notin C_{k}$. Then it follows from the above that $T=l u b_{k} T_{k}$ in $T S . T_{k}$ is called the restriction of $T$ to $I_{k}$ since (by minimality of $T_{k}$ ) only configurations in $I_{k}$ act as a left-hand side. Note also that only configurations in $C_{k}$ act as a right-hand side.

\section{Section 3 Operational Semantics of $\mathscr{B}$}

In this section we introduce the abstract backtracking language $\mathscr{B}$ and present an operational semantics based on a deterministic transition system. $\mathscr{B}$ can be regarded as a uniform version of PROLOG with cut, in that it reflects the control structure of PROLOG. For a program $d \mid s$ in $\mathscr{B}$, the declaration $d$ will induce a transition system $\rightarrow_{d}$ while the statement $s$ induces (given a state) an initial configuration. The operational semantics then is the value of the final configuration (if it exists) of the maximal transition sequence w.r.t. $\rightarrow_{d}$ starting from the initial configuration w.r.t. $s$, 
(3.1) Definition Fix a set of actions Action and a set of procedure names Proc. We define the set of elementary statements EStat $=\left\{a\right.$, fail, $!, s_{1}$ or $s_{2}, x \mid a \in$ Action, $s_{i} \in$ Stat, $x \in$ Proc $\}$, the set of statements Stat $=\left\{e_{1} ; \ldots ; e_{r} \mid r \in \mathbb{N}, e_{i} \in\right.$ EStat $\}$ and the set of declarations Decl $=\left\{x_{1} \leftarrow s_{1}: \ldots: x_{r} \leftarrow s_{r} \mid r \in \mathbb{N}, x_{i} \in\right.$ Proc, $s_{i} \in$ Stat,$\left.i \neq j \Rightarrow x_{i} \neq x_{j}\right\}$. The backtracking language $\mathscr{B}$ is defined by $\mathscr{B}=\{d|s| d \in$ Decl, $s \in$ Stat $\}$.

We let $a$ range over Action, $x$ over Proc, $e$ over EStat, $s$ over Stat and $d$ over Decl. We write $x \leftarrow s \in d$ if $x \leftarrow s=x_{i} \leftarrow s_{i}$ (for some i) or if $s=$ fail otherwise.

Next we give an operational semantics to our backtracking language $\mathscr{B}$. We associate with a declaration $d \in$ Decl a deterministic transition system $\rightarrow_{d}$. The internal configurations of $\rightarrow_{d}$ are stacks. Each frame on a stack represents an alternative for the execution of some initial goal, i.e. statement. As such a frame consists of a generalized statement and a local state. The state can be thought of holding the values of the variables for a particular alternative. The generalized statement is composed from ordinary statements supplied with additional information concerning the cut: Each component in a generalized statement corresponds with a (nested) procedure call. The left-most component is the body being evaluated at the moment, i.e. the most deeply nested one. Since executing a cut amounts to restoring the backtrack stack as it was at the moment of procedure entry, we attach to a statement a stack (or pointer), that constitutes (points to) the substack of the alternatives that should remain open after a cut in the statement is executed. We call this stack the dump stack of the statement, cf. [JM].

(3.2) DEFInITION Fix a set $\Sigma$ of states. Define the set of generalized statements by GStat = $\left\{\left\langle s_{1}, D_{1}\right\rangle: \ldots:\left\langle s_{r}, D_{r}\right\rangle \mid r \in \mathbb{N}, s_{i} \in\right.$ Stat,$D_{i} \in$ Stack, $\left.i<j \Rightarrow D_{i} \geqq_{s s} D_{j}\right\}, \gamma$ denotes the empty generalized statement, the set of frames by Frame $=\{[g, \sigma] \mid g \in$ GStat, $\sigma \in \Sigma\}$ and the set of stacks by Stack $=\left\{F_{1}: \ldots: F_{r} \mid r \in \mathbb{N}, F_{i}=\left[\left\langle s_{1}, D_{1}\right\rangle: \ldots:\left\langle s_{q}, D_{q}\right\rangle, \sigma\right] \in\right.$ Frame s.t. $\left.F_{i+1}: \ldots: F_{r} \geqq_{s s} D_{j}\right\}$ (with $S \geqq s s S^{\prime} \Leftrightarrow S^{\prime}$ is a substack of $S$ ). Let Conf $=$ Stack $\cup \Sigma \cup\{\Omega\}$ be the set of configurations.

Fix an action interpretation $I:$ Action $\rightarrow \Sigma \rightarrow_{\text {part }} \Sigma$, that reflects the effect of the execution of an action on a state. (The language $\mathscr{B}$ gains flexibility if actions are allowed to succeed in one state, while failing in another. Hence we model failure as partiality.) Let $T S$ be the collection of all deterministic transition system with configurations in Conf, internal configurations in Stack, final configurations in $\Sigma$, undefined configuration $\Omega$, domain of values $\Sigma$, valuation $\alpha: \Sigma \rightarrow \Sigma$ with $\alpha(\sigma)=\sigma$. We distinguish $\delta \in \Sigma$ that will denote unsuccessful termination.

(3.3) DEFINITION Let $d \in$ Decl. $d$ induces a deterministic transition system in TS with as step relation the smallest subset of Conf $\times$ Conf s.t.

(i) $\quad \mathbf{E} \rightarrow{ }_{d} \delta$

(ii) $[\gamma, \sigma]: S \rightarrow{ }_{d} \sigma$

(iii) $\quad[\langle\epsilon, D\rangle: g, \sigma]: S \rightarrow{ }_{d}[g, \sigma]: S$

(iv) $\quad[\langle a ; s, D\rangle: g, \sigma]: S \rightarrow{ }_{d}\left[\langle s, D\rangle: g, \sigma^{\prime}\right]: S \quad$ if $\sigma^{\prime}=I(a)(\sigma)$ exists 
$[\langle a ; s, D\rangle: g, \sigma]: S \rightarrow_{d} S$ otherwise

(v) $[\langle$ fail $; s, D\rangle: g, \sigma]: S \rightarrow{ }_{d} S$

(vi) $\quad[\langle! ; s, D\rangle: g, \sigma]: S \rightarrow_{d}[\langle s, D\rangle: g, \sigma]: D$

(vii) $\quad\left[\left\langle x^{\prime} ; s, D\right\rangle: g, \sigma\right]: S \rightarrow{ }_{d}\left[\left\langle s^{\prime}, S\right\rangle:\langle s, D\rangle: g, \sigma\right]: S \quad$ if $x^{\prime} \leftarrow s^{\prime} \in d$

(viii) $\quad\left[\left\langle\left(s_{1}\right.\right.\right.$ or $\left.\left.\left.s_{2}\right) ; s, D\right\rangle: g, \sigma\right]: S \rightarrow F_{1}: F_{2}: S \quad$ where $F_{i}=\left[\left\langle s_{i} ; s, D\right\rangle: g, \sigma\right](i=1,2)$

We comment briefly on each of the above transitions (more precisely transition schemes): (i) The empty stack, denoted by $\mathrm{E}$, has no alternatives left to be tried. Hence the computation terminates unsuccessfully yielding $\delta$. (ii) If the top frame contains the empty generalized statement, denoted by $\gamma$, the computation terminates successfully. The local state $\sigma$ of the frame is delivered as result. (iii) If the left-most component of a generalized statement has become empty (as is the case when a procedure call or the initial statement has terminated), i.e. has format $\langle\epsilon, D\rangle$, the statement-dump stack pair is deleted from the frame. The computation continues with the remaining generalized statement. (iv) In case an action $a$ in the top frame has become active, the action interpretation $I$ is consulted for the effect of $a$ in $\sigma$. If $I(a)(\sigma)$ is defined the state is transformed accordingly. If $I(a)(\sigma)$ is not defined the frame fails and is popped of the stack. (v) Execution of fail amounts to failure of the current alternative. Hence the top frame is popped of the backtrack stack. Control is transferred to the new top frame. (vi) The transition concerning the cut represents removal of alternatives; the top frame continues its execution. Since the dump stack $D$ is a substack of the backtrack stack $S$, replacing the backtrack stack by the current dump stack indeed amounts - in general - to deletion of frames, i.e. of alternatives. (Note that the right-hand stack is well-formed by definition of GStat.) (vii) A call initiates body replacement. The body is looked up in the declaration $d$ and becomes the active component of the generalized statement in the top frame. This component has its own dump stack, which is (a pointer to) the backtrack stack at call time. (viii) Execution of an alternative composition yield two new frames: an active frame corresponding to the left component of the or-construct and a suspended frame corresponding to the right component.

(3.4) DEFINITION The operational semantics $\mathscr{O}: \mathscr{B} \rightarrow \Sigma \rightarrow \Sigma_{\perp}$ for the backtracking language $\mathscr{B}$ is defined by $\mathscr{O}(d \mid s)(\sigma)=\alpha_{d}([\langle s, \mathbf{E}\rangle, \sigma])$ where $\alpha_{d}$ :Conf $\rightarrow \Sigma_{\perp}$ is the valuation associated with the deterministic transition system induced by $d$.

\section{Section 4 Denotational Semantics for $\mathscr{B}$}

By now a standard approach has been established for defining a denotational semantics of a sequential procedural language. Cf. [MS], [St], [Ba1]. We show that a semantics of $\mathscr{B}$ in this section and PROLOG in section 6 can also be given along these lines. Standard semantics uses environments and continuations.

Environments are needed because the denotation $\mathbb{I} s \Pi_{\mathfrak{a}}$ of a statement $s$ depends amongst others on the meaning of the procedure names occurring in $s$. Therefore the function II $-I_{\alpha}$ takes an environment $\eta \in E n v$ as a parameter which defines the meaning of all procedure names.

The flow of control will be described using continuations. For languages like PASCAL, 
where flow of control is not very intricate, a denotation $\left[\mathbf{s} \Pi_{\downarrow}\right.$ needs only one continuation as a parameter. Languages containing backtrack constructs, like SNOBOL4, are best described using two continuations, cf. [Te]. In order to capture the effects of the cut operator yet another continuation will be needed. (As is observed independently by M. Felleisen in [Wi], p. 273.) In order to explain how these continuations will be used we introduce them one after another. First we shall discuss the PASCAL-subset of $\mathscr{B}$, i.e. $\mathscr{B}$ without or , fail and cut $!$. Thereafter we shall examine the SNOBOL4-subset of $\mathscr{B}$, introducing the $\underline{\text { or }}$ and fail constructs, and finally we shall explain how all three continuations are used in describing full $\mathscr{B}$.

In order to understand the essence of continuation semantics, consider a substatement $s$ that is part of a statement $s^{\prime}$ (in the PASCAL-fragment of $\mathscr{B}$ ). The denotation [ $s \Pi_{s}$ will be a function that will, in the end, deliver an answer in $\Sigma_{\perp}$. This answer is not the result of executing $s$ alone, but the result of evaluating the whole statement $s^{\prime}$ of which $s$ is a substatement. Therefore the result does not only depend on an environment $\eta$ and an initial state $\sigma$, it also depends on a denotation $\xi$ of the remainder of the statement, to be executed once evaluation of $s$ has terminated. This leads to the following functionality of $\mathbb{I} \cdot \mathbb{1}_{\mathbb{1}}:$ Env $\rightarrow$ Cont $\rightarrow \Sigma \rightarrow \Sigma_{1}$. Here Cont $=\Sigma \rightarrow \Sigma_{\perp}$ since the future $\xi$ of a computation will in the end yield an answer, but this answer depends on an intermediate state, viz. the result of evaluating $s$ alone. A typical clause in our semantics up till now, describing the composition operator ";", will be $\llbracket e ; s \prod_{q} \eta \xi \sigma=$ II $e \prod_{e} \eta\left\{\left[I s \prod_{\mathrm{d}} \eta \xi\right\} \sigma\right.$, which says that the answer obtained by executing $e ; s$ before $\xi$ will be equal to the answer resulting from execution of $e$ before \{ execution of $s$ before $\xi$ \}.

The next stage is to introduce backtracking in the language by adding the constructs or and fail (and by allowing actions to fail). Describing the flow of control is more complicated now. The problem is that the notion "future of the computation" is not that obvious any more. Evaluation of a statement $s$ can terminate for two reasons now. The first one, successful termination, is similar to the situation we had before. In this case the future of the computation is realized by executing the remainder of the statement textually following $s$. But now it is also possible that evaluation of $s$ terminates in failure, e.g. by executing a fail statement. Now the rest of the computation is determined by backtracking to the alternatives built up through execution of or statements in earlier stages of the computation. Such a doubly edged future can best be captured by two continuations, a success continuation $\xi \in S$ Cont and a failure continuation $\phi \in F C o n t$. So now $\left[1 \cdot \mathbb{1}_{\alpha}\right.$ has a new functionality: $\mathbb{I} \cdot \prod_{\alpha}: E n v \rightarrow S$ Cont $\rightarrow F C o n t \rightarrow \Sigma \rightarrow \Sigma_{\perp}$. The meaning II $s \mathbb{I}_{\alpha} \eta \xi \phi \sigma$ of $s$ will depend on $\xi$, denoting the rest of the statement following $s$, and on $\phi$, which is a denotation of the stack of alternatives built up in the past. FCont is best understood by investigating the meaning of the or construct: $\llbracket s_{1}$ or $s_{2} \prod_{d} \eta \xi \phi \sigma=\|\left[s_{1} \prod_{d} \eta \xi \phi^{\prime} \sigma\right.$. This says that executing $s_{1}$ or $s_{2}$ amounts to executing $s_{1}$ with a new failure continuation $\phi^{\prime}$ describing what will happen if $s_{1}$ terminates in failure. In that case $s_{2}$ should be executed, and only if this also ends in failure the computation should proceed as indicated by the original failure continuation $\phi$. Hence we have that $\phi^{\prime}$ equals $\llbracket s_{2} \prod_{\alpha} \eta \xi \phi \sigma$. Combining all this we obtain that II $s_{1}$ or $s_{2} \prod_{\alpha} \eta \xi \phi \sigma$ $=\llbracket\left[s_{1} \mathbb{l}_{a} \eta \xi\left\{\llbracket\left[s_{2} \mathbb{l}_{a} \eta \xi \phi \sigma\right\} \sigma\right.\right.$. Apparently we have FCont $=\Sigma_{1}$. As far as the structure of SCont is concerned, it must be realized that the answer obtained from evaluation of the rest of the statement $s^{\prime}$ does not only depend on the intermediate state resulting from the evaluation of $s$ but 
also on the alternatives built up by executing $s^{t}$ up to and including $s$. For it can very well happen that evaluation of the rest of the statement will terminate in failure. We therefore have SCont $=F$ Cont $\rightarrow \Sigma \rightarrow \Sigma_{\perp}$. We notice that the meaning of the fail statement is straightforward. The answer is the one provided by the failure continuation: II fail $\mathbb{7}_{e} \eta \xi \phi \sigma=\phi$. This is also the case if an action $a$ does not succeed in a state $\sigma$, i.e. $\llbracket a \eta_{e} \eta \xi \phi \sigma=\phi$ if $I(a)(\sigma)$ is undefined, (where $I$ is the fixed action interpretation). If $a$ does succeed the state is transformed according to $I$ and the failure continuation and new state are passed to the success continuation $\xi$. So II $a \rrbracket_{e} \eta \xi \phi \sigma=\xi \phi \sigma^{\prime}$ if $\sigma^{\prime}=I(a)(\sigma)$ exists.

The only construct of full $\mathscr{B}$ that we did not take into account up to now is the cut operator 1. This statement resembles the dummy statement because it does not affect the state. There is a side effect however, since a number of alternatives is thrown away. To be more precise, evaluation of ! discards the alternatives which have been generated since the procedure body in which the ! occurs has been entered. For our semantics this means that evaluation of ! amounts to applying the success continuation to the original state (this is the dummy statement aspect), but also to a new failure continuation. This new failure continuation $\phi^{\prime}$ is in fact an old one, namely the failure continuation which was in effect on entry of the procedure body in which the : occurs. A natural way to obtain this old continuation, which we will call the cut continuation $x \in$ CCont in the sequel, is to provide it as an argument of the meaning function $\left[I-\prod_{a}\right.$. We finally arrive at the functionality $\left[1 \cdot \mathbb{I}_{d}:\right.$ Stat $\rightarrow$ Env $\rightarrow$ SCont $\rightarrow$ FCont $\rightarrow$ CCont $\rightarrow \Sigma \rightarrow \Sigma_{\perp}$, with SCont $=$ FCont $\rightarrow$ CCont $\rightarrow \Sigma \rightarrow \Sigma_{\perp}$ and FCont $=$ CCont $=\Sigma_{\perp}$. The denotation of ! can now be given by II $! \Pi_{e} \eta \xi \phi \kappa \sigma=\xi \varkappa \kappa \sigma$. On entry of a procedure body a new cut continuation has been established. The meaning of a procedure call is straightforward. We have II $x \Pi_{e} \eta \xi \phi \varkappa \sigma=\eta x \xi \phi \varkappa \sigma$, i.e. the arguments $\xi, \phi, \varkappa$ and $\sigma$ are passed to the meaning $\eta x$ of $x$ in the environment $\eta$. The real work is performed in the definition of the environment $\eta$, which should be derived from the declaration $d$ in the program. We want $\eta$ to be a fixed point such that $\eta x$, the meaning of the procedure name $x$ is given by $\eta x \xi \phi \varkappa \sigma=\llbracket s \prod_{0} \eta\{\lambda \bar{\phi} \bar{x}, \xi \bar{\phi} x\} \phi \phi \sigma$ if $x \leftarrow s \in d$. Two effects can be noticed here. First of all a new cut continuation, viz. the failure continuation $\phi$, is "passed", secondly on (successful) termination of $s$ the old cut continuation should be restored and this is captured by passing $\lambda \bar{\phi} \bar{x} \cdot \xi \bar{\phi} x$ instead of $\xi$ to the body $s$.

We now give the denotational semantics of $\mathscr{R}$. We first give the domains: the set failure continuations FCont $=\Sigma_{\perp}$, the set of cut continuations CCont $=\Sigma_{\perp}$, the set of success continuations SCont $=$ FCont $\rightarrow$ CCont $\rightarrow \Sigma \rightarrow \Sigma_{\perp}$ and the set of environments Env = Proc $\rightarrow$ SCont $\rightarrow$ FCont $\rightarrow$ CCont $\rightarrow \Sigma \rightarrow \Sigma_{\perp}$. We denote by $\sigma, \phi, \varkappa, \xi$ and $\eta$ typical elements of $\Sigma$, FCont, CCont, SCont and Env, respectively.

\section{(4.1) DEFINITION}

(i) II $\cdot \mathbb{1}_{e}:$ EStat $\rightarrow$ Env $\rightarrow$ SCont $\rightarrow$ FCont $\rightarrow$ CCont $\rightarrow \Sigma \rightarrow \Sigma_{\perp}$

II $a \rrbracket_{e} \eta \xi \phi \varkappa \sigma=\xi \phi \varkappa \sigma^{\prime} \quad$ if $\sigma^{\prime}=I(a)(\sigma)$ exists

II $a \mathbb{I}_{\boldsymbol{e}} \eta \xi \phi x \sigma=\phi \quad$ otherwise

II fail $\mathbb{1}_{e} \eta \xi \phi * \sigma=\phi$

II ! $\mathbb{I}_{e} \eta \xi \phi \varkappa \sigma=\xi \varkappa \varkappa \sigma$ 
II $s_{1}$ or $s_{2} \prod_{e} \eta \xi \phi \varkappa \sigma=\llbracket s_{1} \mathbb{I}_{\triangleleft} \eta \xi\left\{\llbracket s_{2} \prod_{\alpha} \eta \xi \phi \varkappa \sigma\right\} \varkappa \sigma$

II $x \mathbb{1}_{e} \eta \xi \phi \kappa \sigma=\eta x \xi \phi \kappa \sigma$

(ii) $\mathbb{I} \cdot \mathbb{1}_{\downarrow}:$ Stat $\rightarrow$ Env $\rightarrow$ SCont $\rightarrow$ FCont $\rightarrow$ CCont $\rightarrow \Sigma \rightarrow \Sigma_{\perp}$

II $\epsilon \mathbb{1}_{\mathrm{d}} \eta \xi \phi x \sigma=\xi \phi x \sigma$

II e;s $\prod_{\downarrow} \eta \xi \phi x \sigma=\llbracket e \prod_{e} \eta\left\{\Pi s \Pi_{a} \eta \xi\right\} \phi x \sigma$

(iii) $\Phi:$ Decl $\rightarrow$ Env $\rightarrow$ Env

$\Phi d \eta x \xi \phi x \sigma=\mathbb{I} s \prod_{d} \eta\{\lambda \bar{\phi} \bar{x}, \xi \bar{\phi} x\} \phi \phi \sigma \quad$ if $x \leftarrow s \in d$

(iv) II $\prod_{\mathscr{B}}: \mathscr{B} \rightarrow \Sigma \rightarrow \Sigma_{\perp}$

$\llbracket d \mid s \prod_{\mathscr{B}} \sigma=\llbracket s \prod_{d} \eta_{d} \xi_{0} \phi_{0} x_{0} \sigma$

where $\eta_{d}$ is the least fixed point of $\Phi(d), \xi_{0}=\lambda \phi \kappa \sigma . \sigma$ and $\phi_{0}=\varkappa_{0}=\delta$.

REMARK The least fixed point $\eta_{d}$ defined in 4.1 (iv) can be obtained as the least upperbound of a chain of iterations $\left\langle\eta_{d, i}\right\rangle_{i}$, with $\eta_{d, i}$ defined by $\eta_{d, 0}=\lambda x \xi \phi \kappa \sigma . \perp$ and $\eta_{d, i+1}=$ $\Phi(d)\left(\eta_{d, i}\right)$. From the continuity of $\llbracket \cdot \mathbb{I}_{d}$ we have $\llbracket s \mathbb{\Pi}_{d} \eta_{d}=l u b_{i}$ II $s \Pi_{d} \eta_{d, i}$.

We conclude this section with some comment on the similarity of the operational semantics from the previous section and the denotational semantics of this one. There is a natural correspondence between components of a configuration and the parameters of the denotation of a statement. We compare the answer resulting from evaluation of an elementary statement $e$ and the value obtained from a configuration in which $e$ is about to be executed: II $e \prod_{e} \eta \xi \phi \kappa \sigma$ vs. $[\langle e ; s, D\rangle: g, \sigma]: S$. Here $\xi$ is a denotation of the statements to be executed once $e$ has terminated successfully. So $\xi$ corresponds to the statement $s$ followed by the statements in the generalized statement $g$. The failure continuation $\phi$ is the denotational counterpart of the backtrack stack $S$, the cut continuation $x$ corresponds to the dump stack $D$. It is to be expected that if the correspondence is set up as above, the resulting answers should be the same. This will be formalized in the next section and is pivotal to the equivalence proof given there.

\section{Section 5 Equivalence of $\mathscr{O}$ and $\mathscr{D}$}

In this section we prove the equivalence of the operational and denotational semantics, thus justifying the definition of the latter one.

(5.1) THEOREM For all $d \mid s \in \mathscr{B}: \llbracket\left[d \mid s \mathbb{I}_{\mathscr{B}}=\mathscr{O}(d \mid s)\right.$.

In order to prove theorem 5.1 we use the cpo-structure on the collection of transition systems $T S$ and the continuity of the statement evaluator $\left[I-\mathbb{1}_{\mathfrak{s}}\right.$. According to the remark at the end of section 2 and the remark following definition 4.1 we have that both the operational and denotational semantics can be represented as the limit of a chain, i.e. $\mathscr{O}(d \mid s)(\sigma)=$ $l u b_{i} \alpha_{d, i}([\langle s, \mathbf{E}\rangle, \sigma])$ and $\mathbb{I} d \mid s \mathbb{1}_{\mathscr{B}} \sigma=l u b_{i} \llbracket s \mathbb{1}_{\triangleleft} \eta_{d, i} \xi_{0} \phi_{0} \varkappa_{0} \sigma$.

However, in order to compare $\alpha_{d, i}([\langle s, \mathrm{E}\rangle, \sigma])$ and $\llbracket s \Pi_{d} \eta_{d, i} \xi_{0} \phi_{0} x_{0} \sigma$ we need a stronger result. An intercedent is needed between $\alpha_{d, i}$ and $\mathbb{I} \cdot \mathbb{I}_{\mathfrak{d}}$. We define a (denotational) function 
II - $\mathbb{1}_{\mathscr{C}}$ on (operational) configurations with parameters $d$ and $i$ and show that for all configurations $C$ we have that the value $\alpha_{d, i}(C)$ equals II $C \prod_{\mathscr{C}} d i$. The desired result then follows from II $s \prod_{\sigma} \eta_{d, i} \xi_{0} \phi_{0} x_{0} \sigma=\llbracket[\langle s, \mathrm{E}\rangle, \sigma] \prod_{\mathscr{C}} d i$ which can be checked by routine. Also, perhaps more surprisingly, the equality of $\left[\mathrm{I} \cdot \mathbb{1}_{\mathscr{C}} d i\right.$ and $\alpha_{d, i}$ will be easy to check once the appropriate tool is available.

Having outlined the strategy for the equivalence proof we continue with the definition of the intermediate function II $-\mathbb{I}_{\mathscr{C}}$. First we have to specify the subsets of configurations Stack $_{i}$. The environment $\eta_{d, i}$, being the $i$-th iteration of the bottom-environment, yields the right answer in $x$ provided the call of $x$ leads to at most $i-1$ nested inner calls. This depth of nesting can be controlled in our operational semantics as well. Each component $\left\langle s_{i}, D_{i}\right\rangle$ in a generalized statement $g$ corresponds to a (nested) procedure call. The depth of nesting in $g=\left\langle s_{1}, D_{1}\right\rangle: \ldots:\left\langle s_{r}, D_{r}\right\rangle$ therefore equals r. (Note that, although $g=\left\langle s_{1}, D_{1}\right\rangle: \ldots:\left\langle s_{r}, D_{r}\right\rangle$ we do not require $D_{j} \in$ Stack $_{i}$, this is the case if $[g, \sigma]: S \in S_{\text {tack }}$ since $[g, \sigma]: S \in$ Stack implies that $D_{j}$ is a substack of $S$.)

(5.2) Definition Let GStat $_{i}=\{g \in$ GStat $\mid\|g\| \leqq i\}$ where $\|g\|=r$ for $g=$

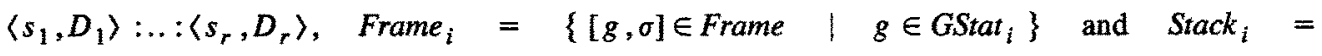
$\left\{F_{1}: \ldots: F_{q} \in\right.$ Stack $\mid F_{j} \in$ Frame $\left._{i}\right\}$.

Next we define the intermediate function $\mathbb{I} \cdot \mathbb{1}_{\mathscr{C}}$. Given a stack $S$ the definition of $\mathbb{I} \cdot \mathbb{1}_{\mathscr{C}}$ can only be elaborated further if $S \in$ Stack $_{i}$. Otherwise the value $\perp$ is returned. Intuitively $\perp$ expresses uncertainty about the value of the configuration. So $\perp$ will be delivered if the elaboration asks for a chain of nested calls of length exceeding $i$.

(5.3) DEFINITION

(i) II $\cdot \mathbb{I}_{\mathscr{C}}:$ Conf $\rightarrow$ Decl $\rightarrow \mathbb{N} \rightarrow \Sigma_{\perp}$

$\llbracket S \rrbracket_{\mathscr{C}} d i=\llbracket S \rrbracket_{\mathscr{F}} d i, \llbracket \sigma \rrbracket_{\mathscr{C}} d i=\sigma, \llbracket \Omega \rrbracket_{\mathscr{C}} d i=\perp$

(ii) II $\cdot \mathbb{I}_{\mathscr{J}}:$ Stack $\rightarrow$ Decl $\rightarrow \mathrm{N} \rightarrow \Sigma_{\perp}$

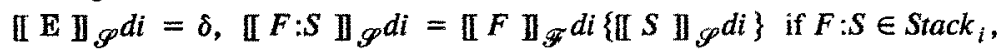

【I $S \rrbracket_{\mathscr{J}} d i=\perp$ if $S \notin$ Stack $_{i}$

(iii) II $-\mathbb{I}_{\mathscr{F}}:$ Frame $\rightarrow$ Decl $\rightarrow \mathbb{N} \rightarrow$ FCont $\rightarrow \Sigma_{\perp}$

$\llbracket[g, \sigma] \rrbracket_{\mathscr{F}} d i \phi=\llbracket[g]_{\mathscr{g}} d i \phi \sigma$

(iv) II $\bullet \mathbb{I}_{g}:$ GStat $\rightarrow$ Decl $\stackrel{\mathscr{T}}{\rightarrow} \mathbb{N} \rightarrow$ FCont $\rightarrow \Sigma \rightarrow \Sigma_{1}$

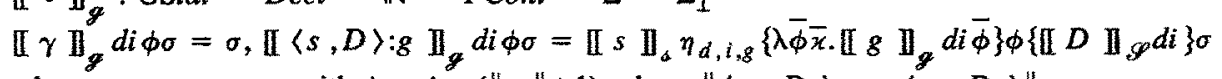

where $\eta_{d, i, g}=\eta_{d, j}$ with $j=i=(\|g\|+1)$ where $\left\|\left\langle s_{1}, D_{1}\right\rangle: \ldots:\left\langle s_{r}, D_{r}\right\rangle\right\|=r$

II $\langle s, D\rangle: g \mathbb{I}_{g} d i$ should yield the right answer only if this can be obtained with less than $i$ nested calls. Now $g$ is responsible for a nesting depth $\|g\|$. So the whole generalized statement $\langle s, D\rangle: g$ has a chain of $\|g\|+1$ nested calls already. This means that $\eta_{d, i, g}$ should allow less then $i-(\|g\|+1)$ calls. (Here - denotes the monus, i.e. subtraction in $\mathbb{N}$.) 
The desired property of the function II $-\prod_{\mathscr{C}}$ is stated in lemma 5.5. First we establish Noetherianity of the restrictions of $\rightarrow_{d}$ to $S_{t a c k}$, i.e. the absence of infinite transition sequences w.r.t. $\rightarrow d_{, i}$. This supplies us with an induction principle that we shall use in the proof of 5.5 .

(5.4) LemMa Let $d \in$ Decl, $i \in \mathbb{N}$ and $\rightarrow_{d, i}$ be the restriction of $\rightarrow_{d}$ to Stack $_{i}$. Then we have that $\rightarrow_{d, i}$ is Noetherian.

PROOF Omitted.

We proceed with the proof of the equality II $\cdot \prod_{\mathscr{C}} d i=\alpha_{d, i}\left(^{*}\right)$. First we notice that this holds for final configurations $\sigma \in \Sigma$, for the undefined configurations $\Omega$, and for internal configurations that admit no transition, i.e. stacks not in Stack $_{i}$.

We shall prove that $\left(^{*}\right)$ is also satisfied by internal configurations that do admit a transition, i.e. stacks in Stack ${ }_{i}$. For this we observe that given the above it suffices to prove: if $C \rightarrow{ }_{d, i} C^{\prime}$ and $\left(^{*}\right)$ holds for $C^{\prime}$ then $\left(^{*}\right)$ holds for $C$ too, by virtue of the Noetherianity of the transition system $\rightarrow_{d, i}$. (This is the principle of Noetherian induction, although in our - deterministic - case it specializes to induction on the length of the maximal transition sequence (which is finite) out of a configuration. See e.g. [Hu].) By definition of the valuation $\alpha_{d, i}$ we have $\alpha_{d, i}(C)=\alpha_{d, i}\left(C^{\prime}\right)$

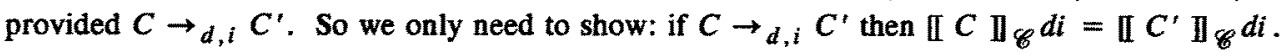

(5.5) LEMMA For all $d \in$ Decl and $i \in \mathbb{N}$ we have $\mathbb{I} \cdot \prod_{\mathscr{C}} d i=\alpha_{d, i}$.

Proof Let $d \in \operatorname{Decl}, i \in \mathbb{N}$ and $C, C^{\prime} \in$ Conf s.t. $C \rightarrow_{d, i} C^{\prime}$. Note $C \in S_{\text {Sack }}$. It suffices to show by structural induction on $C: \llbracket C \prod_{\mathscr{P}} d i=\llbracket C^{\prime} \rrbracket_{\mathscr{C}} d i$.

We only treat case (vi) of definition 3.3: $C=\left[\left\langle x^{\prime} ; s, D\right\rangle: g, \sigma\right]: S$. Say $x^{\prime} \leftarrow s^{\prime} \in d$. We distinguish two subcases: Subcase (a): $\left\|\left\langle x^{\prime} ; s, D\right\rangle: g\right\|=i$. Then we have $C^{\prime}=\Omega$ and $\|g\|=i-1$.

$$
\begin{aligned}
& {\left[\left[\mid\left\langle x^{\prime} ; s, D\right\rangle: g, \sigma\right]: S \prod_{\mathscr{P}} d i\right.}
\end{aligned}
$$

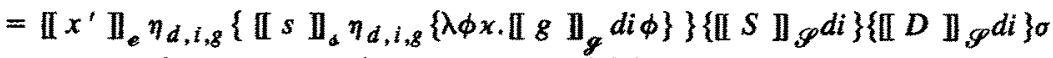

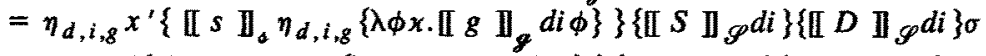

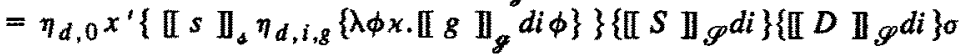

$$
\begin{aligned}
& =\perp \\
& =\llbracket \Omega \rrbracket_{\mathscr{C}} d i \text {. }
\end{aligned}
$$

Subcase (b): $\left\|\left\langle x^{\prime} ; s, D\right\rangle: g\right\|<i$. Then we have $C^{\prime}=\left[\left\langle s^{\prime}, S\right\rangle:\langle s, D\rangle: g, \sigma\right]: S$ and $\|g\|<i-1$.

$$
\begin{aligned}
& \text { [I }\left[\left\langle x^{\prime} ; s, D\right\rangle: g, \sigma\right]: S \prod_{\mathscr{S}} d i
\end{aligned}
$$

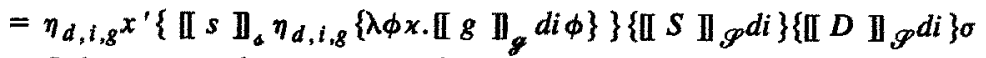

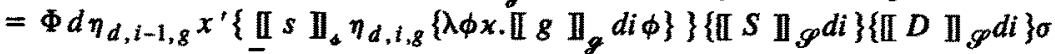

$$
\begin{aligned}
& =\llbracket\left[s ^ { \prime } \prod _ { d } \eta _ { d , i - 1 , g } \overline { \xi } \{ \mathrm { I } s \prod _ { \mathscr { S } } d i \} \left\{\left[\mathbb{S} \mathbb{1}_{\mathscr{P}} d i\right\} \sigma\right.\right.
\end{aligned}
$$

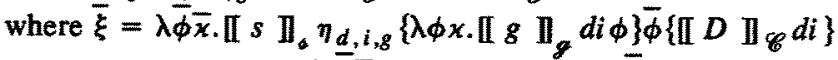

$$
\begin{aligned}
& =\mathbb{I} s^{\prime} \Pi_{\triangleleft} \eta_{d, i,\langle s, D\rangle: g}\left\{\lambda \bar{\phi} \bar{x} . \mathbb{I}\langle s, D\rangle: g \prod_{g} d i \bar{\phi}\right\}\left\{\mathbb{I} S \mathbb{I}_{\mathscr{S}} d i\right\}\left\{\mathbb{I} S \mathbb{I}_{\mathscr{P}} d i\right\} \sigma \\
& \left.=\llbracket\left[\left\langle s^{\prime}, S\right\rangle:\langle s, D\rangle: g, \sigma\right]: S \rrbracket\right]_{\mathscr{S}} d i
\end{aligned}
$$

The other cases are similar, (easier) and omitted here. 
Finally we give the congruence proof of the operational and denotational semantics for $\mathscr{B}$. In the next section we shall modify both this operational and denotational semantics in order to give meaning to PROLOG with cut.

PROOF (of theorem 5.1) Let $\sigma \in \Sigma$. II $d \mid s \prod_{\mathscr{B}^{\sigma}}^{\sigma}=\llbracket s \prod_{a} \eta_{d} \xi_{0} \phi_{0} x_{0} \sigma$ (by definition) =

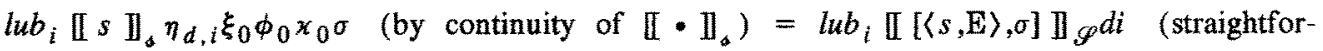
ward) $=l u b_{i} \alpha_{d, i}([\langle s, \mathrm{E}\rangle, \sigma])$ (by the lemma) $=\alpha_{d}([\langle s, \mathrm{E}\rangle, \sigma])$ (by continuity of $\left.\lambda T . \alpha_{T}\right)=$ $\mathscr{O}(d \mid s)(\sigma)$ (by definition).

\section{Section 6 Interpretation of $\mathscr{B}$ into PROLOG}

At the moment PROLOG is probably the most important programming language featuring backtracking. It can be viewed as Horn clause logic with a left-most depth-first computation rule. Nevertheless PROLOG contains execution oriented constructs, e.g. the cut, that makes the standard declarative semantics, that associates to a set of clauses its least Herbrand model ([AE], [EK]), less satisfactorily. Although dating from the early seventies it has lasted until 1984 before a denotational semantics for PROLOG was presented, viz. [JM], that gave account to the behavioural aspects of the language. More recently other (denotational) semantics based on several approaches have appeared, e.g. [DM], [Vi], [Bd]. (See also [Fi], [Fr], [DF], [AB], [BW].)

Our work on the backtracking language $\mathscr{B}$ in the previous sections makes yet another semantics easily available: we can interpret the abstract or uniform statements, declarations and states such that: a set of PROLOG clauses can be regarded as a declaration, a PROLOG goal corresponds with a statement in the abstract language, while a substitution can be viewed as a state. (After all this is not surprising since we designed $\mathscr{B}$ as an abstraction of PROLOG.)

This can be done similarly for the operational semantics. Moreover, the interpretation or deuniformization is done in such a way that the equivalence proof remains valid (after adaptation to minor technicalities). Having factorized the work for a PROLOG semantics in a control flow component (the abstract language $\mathscr{B}$ ) and a logical component (the interpretation of $\mathscr{B}$ towards PROLOG) we obtain presently a congruence proof for the denotational and operational semantics almost for free. Stated otherwise, we have an instance of the "Algorithm = Logic + Control" paradigm $([\mathrm{Kw}])$ at the meta level. (In fact, several semantics of logic programming languages can be considered as generalizations of established models for imperative languages w.r.t. the control; the extensions made are concerned with the particular logic component. Cf. [MR], [GCLS], [Kk], [Ba2]. See in particular [BK] for a related approach in the setting of Concurrent Prolog.)

Unfortunately there is a price to pay for our two pass approach, albeit just a syntactical one. Since we restrict procedure names in $\mathscr{B}$ to have just one procedure body, we can consider clauses with pairwise different head predicates only. We feel free to do so, because this is by no means a computational restriction in the presence of the explicit or-construct and actions interpreted as unifications. (One can use a so called homogeneous form for clauses, as in e.g. [EY], and "or" together clauses with the same head predicate.)

Next we define our variant of the PROLOG language. (Note the similarity with the definition of the language $\mathscr{B}$ in section 3 .) 
(6.1) DEFINITION Let $\mathscr{F}$ be a collection of function symbols, $\mathscr{V}$ a collection of variables and $\mathscr{R}$ a collection of predicate letters. Let Term denote the collection of terms generated by $\mathscr{F}$ over $\mathscr{V}$. Define the set of atomic goals AGoal $=\left\{t_{1}=t_{2}\right.$, fail $, !, G_{1}$ or $G_{2}, R\left(t_{1}, \ldots, t_{k}\right) \mid t_{i} \in$ Term, $G_{i} \in G o a l, R \in \mathscr{R}$ of arity $\left.k\right\}$, the set of goals Goal $=\left\{A_{1} \& . . \& A_{r} \mid r \in \mathbb{N}, A_{i} \in A G o a l\right\}$, true is the empty goal, the set of PROLOG programs Prog $=\left\{A_{1} \leftarrow G_{1}: \ldots ; A_{r} \leftarrow G_{r} \mid r \in \mathbb{N}\right.$, $A_{i}=R_{i}\left(\vec{t}_{i}\right) \in A$ Goal $, \quad i \neq j \Rightarrow R_{i} \neq R_{j}, \quad G_{i} \in$ Goal $\}$. Define PROLOG $=\{P|G|$ $P \in$ Prog , $G \in$ Goal $\}$.

We next develop an operational semantics for PROLOG along the lines of section 3 . In order to obtain a most general answer substitution (i.e. to avoid clashes of logical variables) one is only allowed to resolve an atom against a program clause provided that the variables of the clause are fresh w.r.t. the computation so far. We can achieve this by having infinite supply of copies of the class of variables and tagging every goal with an index that it should be renamed with. (This is in fact structure sharing.) In a global counter we keep track of the number of the first class of variables not used yet.

(6.2) DEFINITION Let Term' be the set of terms generated by $\mathscr{F}$ over $\mathscr{V} \times \mathbb{N}$ and $\Sigma$ be the collection of substitutions over Term', i.e. $\Sigma=\left\{\sigma:\right.$ Term $^{\prime} \rightarrow$ Term $^{\prime} \mid \sigma$ homomorphic $\}$. The set GGoal of generalized goals is defined by GGoal $=\left\{\left\langle G_{1}, D_{1}, m_{1}\right\rangle: \ldots:\left\langle G_{r}, D_{r}, m_{r}\right\rangle \mid r \in \mathbb{N}\right.$, $G_{i} \in$ Goal, $D_{i} \in$ Stack $\left., i \leqq j \Rightarrow D_{i} \geqq_{s s} D_{j}, m_{i} \in \mathbb{N}\right\}$, the set of frames Frame $=\{[g, \sigma, n] \mid$ $g \in$ GGoal, $\sigma \in \Sigma, \quad n \in \mathbb{N}\}$, the set of stacks Stack $=\left\{F_{1}: \ldots: F_{r} \mid r \in \mathbb{N}\right.$, $F_{i}=\left[\left\langle G_{1}, D_{1}, m_{1}\right\rangle: . .:\left\langle G_{r}, D_{r}, m_{r}\right\rangle, \sigma, n\right] \in$ Frame s.t. $\left.F_{i+1}: \ldots: F_{r} \geqq_{s s} D_{j}\right\}$ and the set of configurations Conf $=$ Stack $\cup \Sigma \cup\{\Omega\}$.

The transition system underlying the operational semantics is a straightforward modification of definition 3.3.

Execution of actions $t_{1}=t_{2}$ and procedure calls $R\left(t_{1}, \ldots, t_{k}\right)$ involve unification. We use a black box unification algorithm $m g u$ that yields a most general unifier for two atoms or terms if one exists, and is undefined otherwise. (Cf. [JM], [Fr].) So the effect of the execution of an action $t_{1}=t_{2}$ in state $\sigma$ is the update $\sigma \theta$, i.e. composition of substitutions, of $\sigma$ w.r.t. the most general unifier $\theta$ of $t_{1}$ and $t_{2}$ in state $\sigma$ (and appropriately renamed).

Slightly more deviating is procedure handling, since one has to unify first the call and the head of the particular clause successfully before body replacement can take place. (Stretching a point one may consider PROLOG as a form of conditional rewriting. See also [BW], [EY].) A call is operationally described as follows. Consider a call, i.e. atom, $R\left(t_{1}, \ldots, t_{k}\right)$. First the concerning procedure definition, i.e. clause, is looked up in the declaration, i.e. PROLOG program. Say this is $R\left(\bar{t}_{1}, \ldots, \bar{t}_{k}\right) \leftarrow \bar{G}$. Next we try to unify $R\left(t_{1}, \ldots, t_{k}\right)$ and $R\left(\bar{t}_{1}, \ldots, \bar{t}_{k}\right)$ (considering renaming and the current substitution). If this is possible, i.e. a most general unifier exists, we replace the call by the procedure body, i.e. body of the program clause, extended with dump stack and renaming index, and change the state and global counter according to the side effect, i.e. the result of $m g u$, initiated by the call. We refer the reader to the nice tutorial of [Le] for a discussion on unification in logic programming vs. parameter passing and value return in imperative languages. 
(6.3) Definition Let $P \in$ Prog. $P$ induces a deterministic transition system $\rightarrow_{P}$ with as transition relation the smallest subset of Conf $\times$ Conf s.t.

(i) $\mathrm{E} \rightarrow{ }_{p} \delta$

(ii) $[\gamma, \sigma, n]: S \rightarrow_{P} \sigma$

(iii) [ $\quad$ true $, D, m\rangle: g, \sigma, n]: S \rightarrow_{P}[g, \sigma, n]: S$

(iv) $\left[\left\langle t_{1}=t_{2} \& G, D, m\right\rangle: g, \sigma, n\right]: S \rightarrow_{P}[\langle G, D, m\rangle: g, \sigma \theta, n]: S$

if $\theta=m g u\left(t_{1}^{(m)} \sigma, t_{2}^{(m)} \sigma\right)$ exists

$\left[\left\langle t_{1}=t_{2} \& G, D, m\right\rangle: g, \sigma, n\right]: S \rightarrow_{P} S$ otherwise

(v) $\quad[\langle$ fail \& $G, D, m\rangle: g, \sigma, n]: S \rightarrow_{P} S$

(vi) $\quad[\langle! \& G, D, m\rangle: g, \sigma, n]: S \rightarrow_{P}[\langle G, D, m\rangle: g, \sigma, n]: D$

(vii) $\left[\left\langle R\left(t_{1}, \ldots, t_{k}\right) \& G, D, m\right\rangle: g, \sigma, n\right]: S \rightarrow_{P}[\langle\bar{G}, S, n\rangle:\langle G, D, m\rangle: g, \sigma, n+1]: S$

if $R\left(\bar{t}_{1}, \ldots, \bar{t}_{k}\right) \leftarrow \bar{G} \in P$ and $\theta=m g u\left(R\left(t_{1}^{(m)}, \ldots, t_{k}^{(m)}\right) \sigma, R\left(\bar{t}_{1}^{(n)}, \ldots, \bar{t}_{k}^{(n)}\right)\right)$ exists

$\left[\left\langle R\left(t_{1}, \ldots, t_{k}\right) \& G, D, m\right\rangle: g, \sigma, n\right]: S \rightarrow_{P} S$ otherwise

(viii) $\left[\left\langle\left(G_{1}\right.\right.\right.$ or $\left.\left.\left.G_{2}\right) \& G, D, m\right\rangle: g, \sigma, n\right]: S \rightarrow F_{1}: F_{2}: S$

where $F_{i}=\left[\left\langle G_{i} \& G, D, m\right\rangle: g, \sigma, n\right]$

In the above definition we denote by $t^{(m)}$ the term in Term' obtained by renaming in $t$ variables in $\mathscr{V}$ into the corresponding variables in $\mathscr{V} \times\{m\}$. We use suffix notation for the application and composition of substitutions.

The operational semantics is defined similar to definition 3.4. Here, in the context of logic programming, we choose to fix the start state, viz. the identity substitution $\sigma_{i d}$. The renaming index is set to 1 having used 0 for the top-level goal already.

(6.4) DEFINITION The operational PROLOG-semantics $\mathscr{O}:$ PROLOG $\rightarrow \Sigma_{\perp}$ is defined by $\mathscr{O}(P \mid G)=\alpha_{P}\left(\left[\langle G, \mathrm{E}, 0\rangle, \sigma_{i d}, 1\right]\right)$ where $\alpha_{P}:$ Conf $\rightarrow \Sigma_{\perp}$ is the valuation associated with the transition system induced by $P$.

Having discussed already the idiosyncrasies of PROLOG w.r.t. unification-action and call, it is clear how to adapt the denotational semantics of $\mathscr{B}$ in order to obtain a denotational semantics for PROLOG.

First we redefine the functionality of environments and success continuations. Define Atom $=\left\{R\left(t_{1}, \ldots, t_{k}\right) \mid R \in \mathscr{R}\right.$ of arity $k, t_{i} \in$ Term $\}$. (Atom is the PROLOG-counterpart of Proc.) Let Env = Atom $\rightarrow \mathbb{N} \rightarrow$ SCont $\rightarrow$ FCont $\rightarrow$ CCont $\rightarrow \Sigma \rightarrow \mathbb{N} \rightarrow \Sigma_{\perp}$ and SCont $=$ FCont $\rightarrow$ CCont $\rightarrow \Sigma \rightarrow \mathbb{N} \rightarrow \Sigma_{\perp}$. We take FCont and CCont as defined previously (with $\Sigma_{\perp}$ implicitly changed).

(6.5) DEFINITION

(i) [I $\cdot \mathbb{1}_{\mathscr{A}}:$ AGoal $\rightarrow$ Env $\rightarrow \mathbb{N} \rightarrow$ SCont $\rightarrow$ FCont $\rightarrow$ CCont $\rightarrow \Sigma \rightarrow \mathbb{N} \rightarrow \Sigma_{1}$

【 $t_{1}=t_{2} \prod_{\mathscr{A}} \eta m \xi \phi x \sigma n=\xi \phi x\{\sigma \theta\} n$ if $\theta=m g u\left(t_{1}^{(m)} \sigma, t_{2}^{(m)} \sigma\right)$ exists

II $t_{1}=t_{2} \prod_{\mathscr{A}} \eta m \xi \phi x$ on $=\phi \quad$ otherwise

II fail $\rrbracket_{\mathscr{A}} \eta m \xi \phi \varkappa \sigma n=\phi$ 
II ! I. $\eta m \xi \phi \varkappa \sigma n=\xi \varkappa \varkappa \sigma n$

II $G_{1}$ or $G_{2} \prod_{\mathscr{A}} \eta m \xi \phi x \sigma n=\mathbb{I} G_{1} \mathbb{I}_{\mathscr{G}} \eta m \xi\left\{\left[\mathbb{I} G_{2} \rrbracket_{\mathscr{G}} \eta m \xi \phi x \sigma n\right\} x \sigma n\right.$

II $R(\overrightarrow{(\vec{t})}]_{\mathscr{A}} \eta m \xi \phi x \sigma n=\eta\{R(\vec{t})\} m \xi \phi x \sigma n$

(ii) II $\cdot \mathbb{I}_{\mathscr{G}}$; Goal $\rightarrow$ Env $\rightarrow \mathbb{N} \rightarrow$ SCont $\rightarrow$ FCont $\rightarrow$ CCont $\rightarrow \Sigma \rightarrow \mathbb{N} \rightarrow \Sigma_{\perp}$

II true $\mathbb{I}_{\mathscr{G}} \eta m \xi \phi \varkappa \sigma n=\xi \phi \varkappa \sigma n$

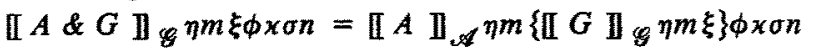

(iii) $\Phi:$ Prog $\rightarrow$ Env $\rightarrow$ Env

$\Phi P \eta\{R(\vec{t})\} m \xi \phi x \sigma n=\mathbb{I} G_{0} \mathbb{1}_{\mathscr{G}} \eta n\{\lambda \bar{\phi} \bar{\varkappa} \cdot \xi \bar{\phi} \varkappa\} \phi \phi\{\sigma \theta\}\{n+1\}$

if $R\left(\vec{t}_{0}\right) \leftarrow G_{0} \in P$ and $\theta=m g u\left(R\left(\vec{t}^{(m)}\right) \sigma, R\left(\vec{t}_{0}^{(n)}\right)\right)$ exists

$\Phi P \eta\{R(\vec{t})\} m \xi \phi \varkappa \sigma n=\phi \quad$ otherwise

(iv) II $\bullet \mathbb{1}_{\text {Frolag }}:$ PROLOG $\rightarrow \Sigma_{\perp}$

【 $P \mid G \prod_{\mathscr{P}_{\mathrm{ralog}}}=\llbracket G \prod_{\mathscr{G} \eta_{P}} 0 \xi_{0} \phi_{0} \varkappa_{0} \sigma_{i d} 1$

where $\eta_{P}$ is the least fixed point of $\Phi(P), \xi_{0}=\lambda \phi x \sigma n . \sigma$ and $\phi_{0}=x_{0}=\delta$

It is a matter of routine to obtain the equivalence of the operational and denotational semantics for PROLOG along the lines of section 5 .

\section{Section 7 References}

[AE]. K.R. Apt and M.H. van Emden, "Contributions to the Theory of Logic Programming," Joumal of the ACM 29, pp. 841-862 (1982).

[AB]. B. Arbab and D.M. Berry, "Operational and Denotational Semantics of Prolog," Journal of Logic Programming 4, pp. 309-329 (1987).

[Bd]. M. Badinet, "Proving Termination Properties of PROLOG Programs: A Semantic Approach," pp. 336-347 in Proc. LICS'88, Edinburgh (1988).

[BW]. J.C.M. Baeten and W.P. Weijland, "Semantics for Prolog via Term Rewrite Systems," pp. 314 in Proc. 1st International Workshop on Conditional Term Rewriting Systems, ed. S. Kaplan and J.-P. Jouannaud, LNCS 308, Springer (1987).

[Ba1]. J.W. de Bakker, Mathematical Theory of Program Correctness, Prentice Hall International, London (1980).

[Ba2]. J.W. de Bakker, "Comparative Semantics for Flow of Control in Logic Programming without Logic," Report CS-R88.., Centre for Mathematics and Computer Science, Amsterdam, to appear (1988).

[BK]. J.W. de Bakker and J.N. Kok, "Uniform Abstraction, Atomicity and Contractions in the Comparative Semantics of Concurrent Prolog," in Proc. FGCS'88, Tokyo (1988).

[BKMOZ]. J.W. de Bakker, J.N. Kok, J.-J.Ch. Meyer, E.-R. Olderog, and J.I. Zucker, "Contrasting Themes in the Semantics of Imperative Concurrency," pp. 51-121 in Current Trends in Concurrency: Overviews and Tutorials, ed. J.W. de Bakker, W.P de Roever \& G. Rozenberg, LNCS 224, Springer (1986).

[BM]. J.W. de Bakker and J.-J.Ch. Meyer, "Metric Semantics for Concurrency," BIT 28, pp. 504-529 (1988).

[BMOZ]. J.W. de Bakker, J.-J.Ch. Meyer, E.-R. Olderog, and J.I. Zucker, "Transition Systems, Metric Spaces and Ready Sets in the Semantics for Uniform Concurrency," Joumal of Computer System Sciences 36, pp. 158-224 (1988).

[Br]. A. de Bruin, Experiments with Continuation Semantics: Jumps, Backtracking, Dynamic Networks, Dissertation, Free University, Amsterdam (1986). 
[DM]. S.K. Debray and P. Mishra, "Denotational and Operational Semantics for Prolog," Jourmal of Logic Programming 5, pp. $61-91$ (1988).

[DF]. P. Deransart and G. Ferrand, "An Operational Formal Definition of PROLOG," Rapport de Recherche 598, INRIA, Rocquencourt (1986).

[EK]. M.H. van Emden and R.A. Kowalski, "The Semantics of Predicate Logic as a Programming Language," Journal of the ACM 23(4), pp. 773-742 (1976).

[EY]. M.H. van Emden and K. Yukawa, "Logic Programming with Equations," Journal of Logic Programming 4, pp. 265-288 (1987).

[Fi]. M. Fitting, "A Deterministic PROLOG Fixpoint Semantics," Journal of Logic Programming 2, pp. 111-118 (1985).

[Fr]. G. Frandsen, "A Denotational Semantics for Logic Programming," DAIMI PB-201, Aarhus University, Aarhus (1985).

[GCLS]. R. Gerth, M. Codish, Y. Lichtenstein, and E. Shapiro, "Fully Abstract Denotational Semantics for Concurrent Prolog," pp. 320-335 in Proc. LICS'88, Edinburgh (1988).

[Hu]. G. Huet, "Confluent Reductions: Abstract Properties and Applications to Term Rewriting Systems," Joumal of the ACM 27(4), pp. 797-821 (1980).

[JM]. N.D. Jones and A. Mycroft, "Stepwise Development of Operational and Denotational Semantics for Prolog," pp. 281-288 in Proc. SLP'84, Atlantic City (1984).

[Kk]. J.N. Kok, "A Compositional Semantics for Concurrent Prolog," pp. 373-388 in Proc. STACS'88, ed. R. Cori \& M. Wirsing, LNCS 294, Springer (1988).

[KR]. J.N. Kok and J.J.M.M. Rutten, "Contractions in Comparing Concurrency Semantics," pp. 317-332 in Proc. ICALP'88, ed. T. Lepistö \& A. Salomaa, LNCS 317, Springer (1988).

[Kw]. R. Kowalski, "Algorithm $=$ Logic + Control," Communications of the ACM 22(7), pp. 424436 (1979).

[Le]. G. Levi, "Logic Programming: the Foundations, the Approach and the Role of Concurrency," pp. 396-441 in Current Trends in Concurrency; Overviews and Tutorials, ed. J.W. de Bakker, W.P de Roever \& G. Rozenberg, LNCS 224, Springer (1986).

[LQ]. J.W. Lloyd, Foundations of Logic Programming, Springer, Berlin (1984).

[MR]. A. Martelli and G. Rossi, "On the Semantics of Logic Programming Languages," pp. $327-334$ in Proc. ICLP'86, ed. E. Shapiro, LNCS 225, Springer (1986).

[MS]. R. Milne and C. Strachey, A Theory of Programming Language Semantics, Chapman \& Hall, London and Wiley, New York, 2 Volumes (1976).

[PR]. G.D. Plotkin, "A Structural Approach to Operational Semantics," DAIMI FN-19, Aarhus University, Aarhus (1981).

[St]. J.E. Stoy, Denotational Semantics - The Scott-Strachey Approach to Programming Language Theory, MIT Press, Cambridge (1977).

[Te]. R.D. Tennent, "Mathematical Semantics of SNOBOL4," pp. 95-107 in Proc. POPL'73, Boston (1973).

[Vi]. E.P. de Vink, "Equivalence of an Operational and a Denotational Semantics for a Prolog-like Language with Cut," Report IR-151, Free University, Amsterdam (1988).

[Wi]. Formal Description of Programming Concepts - III, ed. M. Wirsing, North-Holland, Amsterdam (1987). 